\title{
LAS REPRESENTACIONES DE LOS FUTUROS PROFESORES SOBRE LOS USOS DE LA TECNOLOGÍA EN LA ESCUELA. UN ESTUDIO NARRATIVO
}

\author{
The representations of the future teachers on the uses \\ of technology in school. A narrative study
}

Joaquín PAREDES-LABRA y Ada FREITAS CORTINA

Universidad Autónoma de Madrid. España.

joaquin.paredes@uam.es; adacctorres@gmail.com

bttps://orcid.org/0000-0003-2294-9121; bttps://orcid.org/0000-0002-9731-4641

Fecha de recepción: 21/10/2019

Fecha de aceptación: 01/02/2020

Fecha de publicación en línea: 10/03/2020

\section{RESUMEN}

En un contexto de imposición de la incorporación de tecnologías digitales en los centros educativos, el pensamiento pedagógico de los maestros todavía se presenta disociado de la aplicación de las tecnologías educativas. Este trabajo tiene el propósito de analizar y comprender cómo una propuesta aparentemente benéfica, como la de la utilización de la tecnología educativa en las escuelas, tiene un predicamento marcadamente negativo entre muchos docentes. Esto se pone de manifiesto particularmente en la formación inicial de los maestros. A partir de un enfoque narrativo de la experiencia de los estudiantes de la educación, se trata de identificar el tipo de relato con el que se ha acompañado y que se le ha asignado popularmente a la tecnología en las escuelas, que impide la puesta en marcha de una narración alternativa de las posibilidades de una escuela con tecnología. Una investigación narrativa de carácter cualitativo se pone en marcha aplicando la técnica de grupo focal a una acción educativa donde, junto a grupos de futuros profesionales de la educación, futuros maestros y tecnólogos educativos, se recuperan sus imaginarios de la tecnología en el tipo de 
película comercial que les hace evocar el concepto. Se realizaron tres técnicas de esta naturaleza en otros tantos grupos de clase durante los cursos 18-19 y 19-20 con un total de 73 estudiantes de ambos sexos de una universidad española. Mediante su análisis, se recogen cuatro relatos populares sobre la tecnología educativa: humanista, maquinista, cientifista y futurista. A la luz de estos relatos que se encarnan en diversos imaginarios escolares, basados en la tradición de la propia escuela y la universidad, se pone de manifiesto una nueva narrativa humanista que combina aspectos cientifistas y futuristas, que podría ayudar a repensar las posibilidades de las tecnologías dentro de una educación alternativa.

Palabras clave: Tecnología educativa; uso educativo; representación mental; apropiación tecnológica; narrativa educativa; humanismo; identidad digital; formación inicial del profesorado.

\section{ABSTRACT}

In the context of imposing the incorporation of digital technologies in schools, the pedagogical thinking of teachers still has disassociated from the application of educational technology. The purpose of this work is to analyse and understand how an apparently beneficial proposal, such as the use of educational technology in schools, has such a negative predicament among so many teachers. This is particularly evident in initial teacher training. From a narrative approach to the student experience, it is about identifying the kind of story that has been accompanied and popularly assigned to technology in schools, which prevents the implementation of an alternative narrative of the possibilities of a school with technology. The qualitative narrative research is launched by applying the focus group technique to educational activity, with groups of future education professionals, future teachers, and future educational technologists. Their imaginary of technology is recovered in the type of commercial film that makes them evoke the concept. Three techniques of this nature were performed in as many class groups during courses 18-19 and 19-20, with 73 students, both genres, from a Spanish university. In the analysis, four popular stories about educational technology are collected: humanist, machinist, scientist, and futurist. In the light of these stories that are embodied in various school imaginary, based on the tradition of the school itself and the university, a new humanist narrative that combines scientific and futuristic aspects is revealed, which could help rethink the possibilities of technologies within alternative education.

Key words: Educational technology; educational use; visualization; technology appropriation; educational narrative; humanism; digital identity; initial teacher training.

\section{INTRODUCCIÓN}

El pensamiento tecnológico en los centros educativos se presenta, a veces con cierta ingenuidad, como benéfico. También ha sido calificado como 
"tecno-romanticismo" (Bautista, 1996), que se coloca unas anteojeras para entender la relación de este recurso con la escuela en su conjunto (Murcia, 2012), o como «institución imaginada» (tomando prestado el concepto de "México imaginario» del antropólogo Bonfil Batalla, 1987), porque se imagina la institución escolar articulada con la tecnología, equiparándola en esa relación a la propia tecnología u otras partes de la cultura.

El discurso positivo sobre la tecnología educativa, con alguna incidencia en la conformación de una ciudadanía crítica y la transformación de la educación y la sociedad, ha sido exhibido históricamente por un amplio colectivo de investigadores. Las definiciones, en el contexto español, de algunos académicos como Rodríguez de las Heras (1991), Area (1991) o Cabero (2001) así lo atestiguan.

Los presupuestos educativos de la introducción de la tecnología en las escuelas en los años ochenta parecen referirse a las oportunidades para una sociedad de la información (Masuda, 1984), en un contexto de gran incidencia del desempleo en esa sociedad. La tecnología supondría nuevas oportunidades laborales y, posiblemente, nuevas relaciones sociales en una nueva sociedad. En la siguiente década, de los noventa, aparecen el multimedia digital primero e Internet después. Abrieron un optimista horizonte de acceso al conocimiento con otros códigos y desde cualquier lugar, más información disponible y mejores decisiones (Cebrián, 1998; Trejo, 1996). Incluso se pensó que la ciudad sería más habitable gracias a una toma de decisiones más democrática, facilitada por la tecnología, como ha pasado con los ayuntamientos que han realizado plenos de su corporación retransmitidos por Internet o consultas para decisiones que afectan a la localidad. Simultáneamente, las posibilidades de la educación online fascinaron a muchos (en el contexto español los relevantes trabajos de Salinas, 1998; 1999). Las redes han dominado el nuevo siglo, facilitando la capacidad de comunicarse y construir conocimiento colectivamente (Garrison y Anderson, 2005).

Todas estas bondades fueron pasando al argumentario de los tecnólogos educativos, como aplicaciones posibles en la escuela.

La incorporación de la tecnología educativa en pos de una "escuela tecnológica» es una elaboración más compleja. Supone la comprensión del espíritu y funcionamiento de la escuela, el currículo y los procesos de innovación sostenida. Al respecto, requiere pensar en la finalidad de dicha integración. Ha de tener incidencia en la comunidad (Aranda, Creus y Sánchez-Navarro, 2013) con problemas que afectan a la ciudadanía, estar basada en proyectos (lo que lleva asociado trabajar sobre problemas cotidianos, producir conocimiento y buscar soluciones a problemas) (Hernando Calvo, 2017) y en la colaboración con otros (en la escuela, entre escuelas) (Castañeda, Gutiérrez y Rodríguez Cifuentes, 2011).

La utilización de la tecnología en las escuelas no está exenta de amenazas. Estos mismos autores han avisado de la posible instrumentalización de los docentes o la alienación de los estudiantes, debido a estrategias comerciales, enmascaradas en 
usos transformadores de la educación, como la colaboración, cuyas finalidades son ajenas a los propósitos educativos de la integración educativa de las tecnologías, lo que algunos llaman «uberización» de la educación (http://unescochair.blogs.uoc. edu). Se trata de tendencias neoliberales que también alcanzan al ámbito universitario (Adell, Castañeda, y Esteve, 2018).

A pesar de que la tecnología educativa no tiene, entre sus propósitos, la voluntad de convertir a sus usuarios, los docentes y los estudiantes, en controladores o alienados, se observa un pensamiento pedagógico profundamente anti-tecnológico (Herrán et al., 2012) por parte de los docentes, en particular, y de los centros educativos, en general. Esta forma de pensar la tecnología es una fuente de problemas para su extensión y generalización en las escuelas. Sin embargo, no está siendo analizada en los contextos escolares ni en la formación de los profesionales de la educación. De ahí, conviene revisar la forma en que el pensamiento sobre la tecnología educativa se plantea desde la perspectiva de los futuros maestros y educadores. El propósito es identificar y atajar este grave problema que puede estar en la base de las dificultades de la incorporación de la tecnología educativa en las escuelas.

Para reorientar el diagnóstico de la falta de uso de la tecnología educativa en las escuelas, se hace necesario, por tanto, que la práctica educativa reflexione sobre los relatos de la relación de los docentes con la tecnología digital, con la intención de generar una propuesta educativa ilusionante que permita entrever, a un colectivo mayor de maestros y educadores, que la tecnología educativa puede ser un buen aliado para promover el cambio educativo (Sancho, 2018).

Con el propósito de identificar el relato subyacente entre futuros docentes y otros profesionales de la educación sobre la tecnología, se puso en marcha un estudio cualitativo de enfoque narrativo (Mateos y Núñez, 2011) para indagar la experiencia escolar a través de los relatos del alumnado de la educación. No obstante, conviene recuperar la génesis de los relatos de la relación de los docentes con la tecnología, situando cómo se han construido estos relatos para luego preguntarse hasta qué punto estos imaginarios sociales (Murcia, 2012; Wenger, 1998) han calado entre los futuros docentes, quienes están llamados a construir la escuela del futuro.

\section{Reorientando El diagnóstico de la FALTA DE USO DE LA TECNOLOgía EDUCATIVA EN LAS ESCUELAS}

Frente a la imposición de la incorporación de tecnologías digitales en los centros educativos, las posiciones ingenuas sobre las bondades de la tecnología educativa son percibidas a larga distancia incluso entre los poco avisados. A la lista de transformaciones esperables o «bondades» que esgrimen unos educadores entusiastas, le contestan otros educadores más críticos que le falta adecuación y finura para ajustar aparatos sin alma, ajenos al sistema, a realidades aquejadas de problemas de mayor calado. Y, cuando se insiste en las bondades, la respuesta de la mayoría 
de los maestros y educadores es la lista de agravios producidos por la tecnología en la vida cotidiana (Paredes, Freitas y Andrés, 2017).

Ciertamente, se podría argumentar que el fracaso del uso de la tecnología educativa en las escuelas es cuantitativo, de la escasez del número de centros educativos implicados en la integración de las TIC en la práctica de las escuelas. Porque la cuestión del escaso uso es un primer indicador del desinterés. Así lo ponen de manifiesto estudios sobre la marcha de la introducción de la tecnología educativa en las escuelas españolas de los que cabe recordar, entre otros, los de Escudero (1989), Area (2008) y Paredes, Guitert y Rubia (2015). Estos resultados pueden ser debidos, seguramente, a que las políticas educativas para incorporar tecnologías en las escuelas no parecen haber acertado (Sánchez-Antolín, Ramos y Sánchez Santamaría, 2014).

Sin embargo, el potencial de los imaginarios o relatos (Castoriadis, 1975) del colectivo que conforma la comunidad escolar es fundamental en las reformas y proyectos educativos (Wenger, 1998). Se trata de relatos para un tiempo de narración histórica (Ricoeur, 1995) como un modo de indagar en el conocimiento práctico docente mediante el enfoque narrativo de la investigación educativa (Mateos y Núñez, 2011). Los relatos son parte de las metodologías habituales en el estudio de los procesos de apropiación de la tecnología. Al respecto, las historias de vida y las narrativas personales en general tienen un importante predicamento en la investigación educativa (Duek, Benítez y Moguillansky, 2017).

Un ejemplo de relato triunfante en el ámbito tecnológico de la educación es el Plan Ceibal. A pesar del desinterés generalizado entre los maestros uruguayos (principalmente por falta entendimiento entre actores, y posiblemente por un problema de ajuste del relato a ellos), el entusiasmo despertado por este proyecto entre la población en general fue mayúsculo, como muestran sus equipos de voluntarios organizados y los índices de su aprobación (Fullan, Watson y Anderson, 2013).

Las posiciones críticas, desde el propio movimiento de introducción de tecnologías educativas en las escuelas, alertan contra la simplicidad y proponen una escuela renovada, pero no parecen ser escuchadas, tal vez por la complejidad de los planteamientos que las acompañan (Sancho y Fernández, 2016), tal vez por la falta de un relato colectivo. La poca seducción que ejerce el pensamiento crítico en relación con la tecnología educativa que se pone en marcha en las escuelas no hace corregir el «dadaismo» tecnológico ni convence a quienes parecen imbuidos por otros relatos. Entre medias se encuentran otros relatos eclécticos, una mezcla de "mindfulness", creatividad participativa y nuevo managerialismo, por ejemplo, la propuesta de innovación tecnológica de la educación recopilada por Hernando Calvo (2017).

No obstante, el gran problema es un pensamiento pedagógico profundamente anti-tecnológico, incluso de parte de la academia. En un estudio emprendido con profesores del ámbito de la pedagogía y miembros de los movimientos de renovación 
pedagógica (Herrán et al., 2012), se observó que la mayoría de los docentes no tenían interés o atribuían un carácter alienante a la tecnología. En todo caso, algunos concedían que pudiera ser un auxiliar, pero no un eje central en la materia. Los epítetos que se le dedican a la tecnología y, por extensión, a la tecnología en la escuela son: bombardeo, estupidez, irracionalidad, hipnotismo, fascinación, seducción, manipulación, sesgo, politización, mercantilización, condicionamiento de cabezas, subdesarrollo, adicción, simplificación, falta de diálogo, injusticia y plagio. Salvando los epítetos más destructivos, se adivina una visión secundaria, de acompañamiento, que es la que precisamente convierte las tecnologías en una herramienta para el control. Se postulan otras posiciones, transformadoras, pero no tienen acogida entre los académicos del estudio.

Entonces, si la élite de académicos que orienta a los maestros que realizan el cambio (incluso algunos de los maestros influyentes entre los influyentes) es tan refractaria a la tecnología, el posible cambio educativo con tecnología es prácticamente imposible. Se ha generado, dicho en los términos de la postmodernidad, un relato de control por parte de la tecnología, que no atrae en absoluto a quienes quieren promover cambios en las escuelas, que serían los principales promotores de una escuela alternativa, los aliados naturales, en el sentido gramsciano de Jarpa (2015), de los tecnólogos educativos.

De ahí, conviene realizar una revisión de la génesis de los relatos de la relación de los docentes con la tecnología, para recuperar cómo se han construido los cuatros relatos más populares sobre la tecnología educativa, a saber: el relato humanista, el relato maquinista, el relato cientifista y el relato futurista; y estudiar la articulación de estas dimensiones generales en el imaginario de los futuros docentes.

\subsection{El relato bumanista}

El humanismo, que devuelve la creencia en el ser humano y sus posibilidades, es profundamente universitario (Rico, 1993). Por ello, aúna ciencia, técnica e inteligencia, se convierte en una amenaza contra el statu quo, está interesado no solo por el conocimiento, sino por la forma en que ganarlo y relacionarse con él, modela el mundo, lucha contra la intransigencia y el poder, y esta forma de relacionarse con el conocimiento pervive, con mayor o menor fortuna, hasta nuestros días, a pesar de la especialización. Es tanto un plan para el conocimiento de todo como para la reforma de las sociedades. Leonardo Da Vinci es un icono que representa bien este deseo, descollando en lo que ahora se llamaría lo científico, artístico y técnico.

Le interesa lo que, en lenguaje coloquial, se llamaría "cultura», el acervo acumulado de ideas y artefactos que han legado los antepasados. En ese sentido, está vinculado a lo que, en el lenguaje contemporáneo, se denominaría "cultura del remix", la articulación de un pensamiento basado en la autoridad de los clásicos. La imagen que resume esta posición epistemológica está en el techo de la biblioteca 
de Montaigne en su castillo de la Dordoña, cuya viguería está repleta de citas en griego y en latín.

Los humanistas, como universitarios, se prodigan en intercambios, y viajan por Europa. Su afán de conocer y dialogar los convierte, también, en activistas de su propia forma de comprender el mundo. Escribían en la lengua común del momento y tenían la visión del científico con cultura. La imprenta se convierte en una aliada. Gracias a ella muchos conocen el trabajo de Erasmo y otros.

El humanista y, por extensión, sus estudiantes son hombres libres y críticos, con los rasgos de la universidad que tendrán que esperar tener derecho a expresarse al siglo XIX, porque este pensamiento libre es una amenaza para el sistema. Mientras llega, algunos de ellos, como Galileo o Servet, sufren la venganza del integrismo y el poder preocupado por esa independencia y afirmación de singularidad. El humanista no subordina lo religioso a lo humano, aunque varios siglos más tarde un pensamiento que se reclama humanista enmascare un rearme humano de la religiosidad (Navarro-Daniels y Paukner, 2004).

El sueño humanista se desbaratará cuando los «no latinos» impulsen la ciencia. Al final del siglo XVII, particularmente en Inglaterra y Francia, comienza un proceso de especialización del conocimiento y consolidación de disciplinas científicas independientes, con sus propios métodos y cosmovisión (Ordóñez, Sánchez Ron y Navarro, 2007).

Por todo ello, se plantea que en la cultura humanista lo tecnológico está profundamente cruzado con la cultura contemporánea, tal y como Heiddeger recuerda a Sartre (Molinuevo, 2004; Da Rosa y Trevisan, 2016).

Jugando con los imaginarios sociales (Castoriadis, 1975), convendría reivindicar algunos iconos para conseguir aproximarse a lo que se propone. Una poderosa imagen de la educación humanista contemporánea está, más que en el valioso "Manual del perfecto gentilhombre» de Castiglione, en la película "El club de los poetas muertos", dirigida por Peter Weir, con rasgos tales como la necesidad de apasionarse con el conocimiento, vivir una vida indisociable de la cultura, mantener una posición propia, defender unos ideales y desafiar el poder establecido. Se trata de la relación con la cultura preferida por las escuelas y la universidad. Un mundo en el que parece que las máquinas no tienen nada que aportar.

No obstante, ¿puede la tecnología educativa acompañar o dar respuesta a estos intereses de una escuela humanista? Es bastante posible. En la película "La guerra de las galaxias", el androide $C-3 P O$ representa un pozo de sabiduría fundamental para resolver multitud de dificultades. De hecho, es el asistente y maestro de los niños que tendrán tanta importancia en la saga. Reúne, además, otros rasgos que lo humanizan, como cierta pedantería, la envidia, el rigor y el celo.

De vuelta a las escuelas, existen las que han construido un ideario educativo que, aprovechando las oportunidades de acceso al conocimiento y producción de 
este que traen las tecnologías, involucra a sus estudiantes en proyectos sociales y críticos sin renunciar a un gusto por el acervo cultural.

\subsection{El relato del maquinismo}

Los ordenadores son máquinas. Algunos, con matices, los perciben en ese ámbito. El fabrilismo europeo del siglo XIX, el origen del maquinismo como imaginario o relato, como muestran las novelas de Dickens o Zola, es denigrante para las personas, dejando a la humanidad y sus identidades (que forman parte del ideal humanista) destruidas, junto al medio ambiente.

Este ambiente del fabrilismo ha sido bien recreado en el cine por películas como la saga "Sherlock Holmes», dirigida por Guy Ritchie, que refleja con particular crudeza el origen imperial del movimiento, así como la explotación y dominación del mundo con el final de las culturas locales.

Existe además una estética proveniente de lo que se podría llamar un «integrismo maquinista", cultivada por el fascismo y el estalinismo. A estos totalitarismos les viene bien convertir a las personas en parte de un engranaje, por lo que el imaginario de la eficiencia de las máquinas le resulta muy apropiado a su propaganda, en ejemplos como el movimiento artístico futurista italiano y español, el "coche del pueblo» o Volkswagen y la proeza técnica y estética del metro de Moscú. Son muestras de la preocupación de los totalitarismos por convencer a la gente corriente, las masas, con soluciones para las masas, tal y como plantea Claramonte (2012). Pero estos movimientos trabajan desde guerras y violencia para imponerse como razón de ser, como muestra el trabajo de Bernal (2016) sobre la alianza del conglomerado industrial alemán con el fascismo de los años 30. No es un problema estético, sino la amenaza que suponen y que la ciudadanía percibe.

Como "contra-imaginarios", se han movilizado la pintura y el cine. Así, el cuadro "Guernica", de Pablo Ruiz Picasso, refleja los horrores de la guerra. El maquinismo es profundamente anti-humano y debe estar bajo permanente vigilancia. El maquinismo desemboca en la guerra y el terror. Las máquinas conspiran contra la paz, parece denunciar el pintor. Por su parte, el cine denuncia situaciones denigrantes. Así, Charles Chaplin, en "Tiempos modernos", presenta una desternillante y lúcida crítica de las relaciones laborales. Y en "Neo-Tokio", la película "Blade runnen", dirigida por Ridley Scott, se muestra un medioambiente destruido y la necesidad de una respuesta ambiental sostenible.

Con estos imaginarios se pone en marcha todo un estado de ánimo contra las máquinas. En algunos casos, la irrupción de las máquinas tiene resonancias ludditas (Hobsbawm y Rudé, 1969), pues son vistas como enemigas de los trabajadores menos cualificados, traen el desempleo y conducen a la miseria de las comunidades donde se imponen. En otros, se denuncian los efectos alienantes del maquinismo en la identidad de la ciudadanía de ahora mismo (como plantea Gergen, 1991), 
generando un estado de prevención soportado por noticias alarmantes en los medios de comunicación.

Las condiciones del capitalismo contemporáneo son bien distintas de las que armaron, sustentaron y desarrollaron el fabrilismo del siglo XIX. Como ha explicado Castells (1997-2005), en la segunda mitad del siglo pasado se conformó un modo de producir y distribuir bienes que conformó nodos y redes. Estos nodos se conectaron tecnológicamente con los adelantos del momento, y se aceleró todo con la llegada de Internet. Fue tan importante la configuración de la forma de hacer capitalismo como la aparición de las redes, que se retroalimentaron. La tecnología se ha convertido en una herramienta para el control del consumo mundial. En el imaginario social (aunque con un papel diferente al que, posiblemente, se le atribuye), la tecnología no es ajena a una consecuencia contemporánea del maquinismo, el consumismo.

Un caso especial de consumismo es el "novismo». Es bastante común que los medios que publicitan la tecnología vivan presos del mismo, pues condiciona la agenda tecnológica. El novismo, por su naturaleza, no puede tener relato. Y si lo tiene, solo puede estar referido a su carácter de novedad, al marketing de lo nuevo o, peor aún, al relato que lo asocia a un consumismo estéril, lo que, a su vez, lo coloca en el relato maquinista. Intentar sustentar la integración de la tecnología en las escuelas en el novismo es, además, vincular un cambio a formas de producción colonialistas y alienantes, cuando no simplemente a un vacío argumental.

Con estos antecedentes, ¿tiene alguna posibilidad el maquinismo para alentar el cambio en educación? Posiblemente ninguna, por llevar aparejados la violencia, la alienación o el consumismo. Por si no fuera suficiente argumento el relato maquinista recuperado, la representación del maquinismo podría ser la novela de Dickens, "Oliver Twist", un huérfano de mineros que viven en condiciones pésimas. Nada que ofrecer frente a la educación roussoniana, que se vincula con la naturaleza y formas de libertad.

\subsection{El relato del cientifismo}

El lanzamiento del satélite «Sputnik», un sofisticado producto científico de los años cincuenta, se convirtió en un poderoso imaginario que desafió a los sistemas educativos de Occidente, particularmente a los Estados Unidos. ¿Cómo era posible que la nación atrasada que lo ponía en órbita consiguiera un logro científico tan importante, un artefacto a miles de kilómetros de la Tierra? Parecía un problema de calidad de sus científicos y, por ende, de educación.

Conviene señalar, no obstante, que el imaginario cientifista se venía arropando por los artistas, quienes difundían la importancia de la ciencia en el futuro de los pueblos. Así ocurre con muralistas latinoamericanos como Diego Rivera ( El hombre controlador de universos", 1934, Palacio de Bellas Artes, México).

La educación era interpelada en Occidente para mejorar y hacerse más científica, tanto en sus contenidos como en las formas racionales de ser organizada. 
Algunos rasgos del cientifismo como relato que se quieren destacar son: el sesgo hacia la primacía de la ciencia productiva, cierta atribución de rasgos trascendentes, hablándose de la posibilidad de una nueva "creación", el sesgo de la ciencia aplicada a determinadas lógicas de producción, la generación de una ética científica denominada "transhumanista" y la convivencia con una ética que se podría calificar como "arrepentida" para sustentar el cientifismo. En este sentido, parece que cuando los ciudadanos perciben máquinas tales como ordenadores como una extensión de progreso científico, estos rasgos, y su crítica, acaban formando parte del imaginario cientifista.

Entonces, un problema del cientifismo, como relato dominante, es la atribución de primacía a una forma de hacer ciencia, de dar valor solo a aquella ciencia que sirve a los intereses de una nación, tal y como puede contemplarse habitualmente en la convocatoria de financiación de investigación en áreas prioritarias para un país y por un periodo plurianual. Lo biotecnológico y lo tecno-energético suele formar parte de ese ranking particular de la ciencia (por ejemplo, como argumenta Giachi, 2017). Esta forma de prelaciones tiene efectos en todos los ámbitos de la vida, de tal forma que, al cabo, solo es ciencia lo que se valora como más relevante para ser financiado como ciencia. Esta parte por el todo de la ciencia, esta metáfora que convierte en ciencia determinadas áreas de algunas disciplinas, está embarcada en procesos que asemejan una nueva "creación": tejidos sintéticos, prótesis, inteligencia, vida, etc. Aquí empieza el problema para la ciudadanía. Esa ciencia, de gran poder, no parece tener controles ni límites. ¿Ante quién responderán si se traspasan los intereses de la sociedad, en favor de qué pequeño grupo, o para qué determinados problemas o lógicas de producción actuarán? La imagen de la ciencia, y sus máquinas, tomando el mundo es preocupante.

El propio cientifismo promueve respuestas éticas para estos y otros interrogantes. En este sentido, cabe destacar el movimiento "transhumanista", con sus vertientes de la ciencia para apoyar el hedonismo (David Pearce, https://www.hedweb.com/ confile.htm) y de ética del perfeccionamiento humano (Nick Bostrom, https://nickbostrom.com/). Desgraciadamente, los imaginarios de estas éticas son terroríficos: una humanidad volcada a la inactividad apoyada por la técnica, o una humanidad, aunque hibridada con la técnica, con parecidos a la que refleja la novela distópica «Un mundo feliz» de Huxley (1932), que levantan preocupaciones por los referentes que ofrecen. La tecnología tiene un papel muy poco halagüeño en el enfoque cientifista.

Son muchos los casos de terribles consecuencias para la gente y el planeta derivadas de usos inadecuados de la tecnología, y la formación de estos profesionales y científicos debe articular alguna respuesta. Se trata de una posición ética que intenta que los científicos tomen conciencia de las consecuencias sociales de sus propuestas. Diversos profesionales, como los ingenieros, incluyen en su currículo una formación en la dimensión ética de los efectos de sus proyectos (como reflejan diversos números de una revista clásica sobre la temática, "Science and 
Technology Ethics and Policy"). Estas propuestas son conocidas como enfoque ciencia-tecnología-sociedad (por ejemplo, la propuesta clásica de Baram, 1971). Un representante de este enfoque, de enorme peso en el campo de las tecnologías, es Luciano Floridi (2006). Para este autor, en vez de una entidad ajena (que ofrezca un referente moral, del bien y el mal), serán la calidad de la información y la entropía los responsables de la marcha de los acontecimientos mediados por tecnología. A pesar de esta propuesta, los efectos prácticos (que podrían ayudar a atenuar la asociación del cientifismo con formas de alienación humana) son, de momento, escasos, por las dificultades de operativizarlo.

El cientifismo, como se avanzó, apuesta por la educación. Ahora bien, las premisas de eficiencia y control no son las mejores compañeras para cambiar el imaginario de las máquinas en las que se pretenden apoyar las soluciones ofrecidas (por ejemplo, enseñanza asistida por ordenador).

Dicho todo esto, existen alternativas cientifistas profundamente sociales. En la escuela es posible implementar experiencias de colaboración entre ciencia y escuela, como la que describen Senabre, Ferrán-Ferrer y Perelló (2018). Así se propone la propuesta alfabetizadora de Bruce, Peterson Bishop y Budhathoki (2014). Las máquinas vienen a ayudar a que las personas salgan de los guetos y se acerquen a las universidades. También los laboratorios ciudadanos, parte del denominado "movimiento makem, como el "MediaLab Prado". Uno de sus promotores, Antonio Lafuente (http://old.medialab-prado.es/article/entrevista_con_antonio_lafuente), propone aplicar la lógica de la ciencia (y sus desarrollos e innovaciones) en estrategias de participación ciudadana, por lo que dota de implicaciones políticas estas transformaciones. Tal y como plantea el autor en una entrevista de 2014 (https:// youtu.be/nX1_SvuIw2U), «la tecnología que destruye el planeta quizá nos permita salvarlo".

Este diálogo con la sociedad puede que ayude a las posiciones cientifistas a generar un cambio de imagen de la ciencia y, por ende, de la tecnología. Pero el imaginario está, para los más mayores, en la guerra fría, el botón nuclear y Chernóbil. Y los más jóvenes viven con preocupación el poder del «big data" (Gértrudix, Borges y García, 2017). Así que las máquinas en las escuelas tienen dificultades para sobreponerse al relato del cientifismo.

\subsection{El relato del futurismo}

El fabrilismo y el cientifismo hicieron despertar la imaginación sobre los resultados del progreso indefinido de las sociedades. Al respecto, es muy oportuno consultar una excelente colección de imaginarios que se encuentra en la web "Paleofuture" (https://paleofuture.com). Muchos son hilarantes y, algunos, preocupantes.

Pero, quizá, quien mejor representa la idea de un futuro diferente es el escritor Julio Verne. Sus novelas harán soñar a varias generaciones con posibilidades (que en los siguientes 100 años se realizan) de bienestar de la humanidad. 
JOAQUÍN PAREDES-LABRA Y ADA FREITAS CORTINA

LAS REPRESENTACIONES DE LOS FUTUROS PROFESORES SOBRE LOS USOS DE LA TECNOLOGÍA EN LA ESCUELA. UN ESTUDIO NARRATIVO

De alguna forma, la novela futurista o de anticipación será re-inventada por la de ciencia-ficción, que conformará un relato que pesa poderosamente sobre los usos de la tecnología en la educación. Autores como Isaac Asimov o Ray Bradbury plantearán personajes y universos desconocidos en los que los problemas de humanos y máquinas se desenvuelven.

De forma simultánea, y con argumentos más ligeros, la televisión será el vehículo para proponer un relato visual del futuro, con series como «Star Trek", creada por Gene Roddenberry. Como en las obras de Verne, las novelas de ciencia-ficción y, quizá más claramente, las series televisivas propondrán en numerosas ocasiones argumentos donde la razón y las emociones, tan humanos, serán mejor solución que la fuerza o las prestaciones de las tecnologías (Garci, 1971). Al igual que hacen los capitanes Nemo, Kirk o Picard, con inteligencia y sentimiento, y ayudados por la tecnología, se resolverán las peores crisis. La publicidad de la tecnología escolar de los ochenta y noventa se apoyó en esa imagen futurista para ofrecer mejorar los problemas, en este caso de la educación, gracias a los ordenadores.

Pero esa publicidad, propia del optimismo de los sesenta edulcorado por las series futuristas de la televisión, convivió con una profunda crisis económica y una guerra fría en sus peores momentos en los ochenta. El imaginario futurista evolucionó al mundo cíborg, lleno de problemas, en el que una nueva raza reclama sus derechos a la existencia. Se dibuja un nuevo futuro que no es tan luminoso. Sin embargo, en ese futuro hay una reivindicación de lo profundamente humano desde lo post-humano. El «Manifiesto cíborg», de Donna Haraway (1984, 2016), es una reivindicación de la incompletitud humana desde la reivindicación de los hibridados con tecnología, algo que la propia autora calificó de broma académica, pues lo que se estaba proponiendo, en realidad, es la defensa de múltiples identidades superpuestas en una persona. Quizá lo exprese la actual película «La forma del agua", dirigida por Guillermo del Toro. Puede representar la preminencia de la incompletitud humana, la importancia del sentimiento y la necesidad del respeto al diferente, en un contexto de guerra, explotación e híper-tecnificación.

En los noventa, se propondrá un pensamiento político que reivindica a colectivos específicos a los que se pisotea sus derechos y para quienes se articula un pensamiento que les puede proteger, desde posiciones como el ecologismo, el feminismo, el anticolonialismo o el pensamiento "queen, que perviven en la actualidad en iniciativas tecnológicas tales como "Barbijaputa», "Bitch media» o "Critical media project. Se persigue el ciberactivismo y el empoderamiento de la población más vulnerable (Cerbino y Belotti, 2016; Saura, Muñoz-Moreno, Luengo-Navas y Matos Ortega, 2017).

Con la tecnología futurista, armada de pensamiento postmoderno, se anima a pensar una escuela que superará sus peores pesadillas del pasado (transmisión, uniformización, violencias) con acción política, inteligencia y emociones. Sin embargo, este mensaje es particularmente difícil de aceptar, en la medida en que 
la tecnología es de los poderosos. Y cuando es escuchado, como ha ocurrido con la idea del "ordenador de 100 dólares", lo ha sido por algunos pocos gobiernos de países en dificultades, reunidos alrededor del movimiento de un ordenador por niño (olpc.org).

\section{MeTOdología}

Para identificar el tipo de relato con el que se ha acompañado y que se le ha asignado popularmente a la tecnología educativa en las escuelas, que puede estar impidiendo la puesta en marcha de una narración alternativa de las posibilidades de una escuela tecnológica, se realizó un estudio empírico, de carácter cualitativo, a partir de un enfoque narrativo de una actividad educativa propuesta a los estudiantes y futuros profesionales de la educación en la que se les hace recuperar sus imaginarios sobre la tecnología con la elección de una película comercial que evoque tal concepto.

A partir de la aplicación de la técnica de grupo focal, se solicitó a futuros maestros y futuros tecnólogos educativos que enunciaran cuál era la película comercial que evocaban al sugerírseles el concepto de "tecnología», para hacer aflorar la carga cultural, emotiva y social de este concepto.

Esta técnica se basa en la idea de que la representación de un fenómeno complejo, como es la incidencia de dispositivos tecnológicos en la vida cotidiana de la gente, podría encontrar un imaginario con el que relacionarse en otras representaciones culturales, como son las películas comerciales de una temática determinada. Identificar un relato como representativo de una narrativa puede ser una aproximación al estudio propuesto.

Al igual que pasa con las técnicas proyectivas, las que hacen hablar de las emociones y sentimientos profundos, esta técnica no debería tener valor por sí misma si no se combina con otras pruebas y actividades. Así que la evocación se completa con un debate posterior con el grupo, a modo de grupo focal, sobre el significado de las respuestas ofrecidas, el valor de las mismas para cada uno de los participantes y la capacidad para valorar su percepción del alcance de la tecnología educativa en sus prácticas profesionales.

La aclaración del sentido de las películas elegidas mayoritariamente por el grupo conlleva una visión de la sociedad y sus problemas, con lo que se ubica la perspectiva con la que se han elegido estos filmes para representar una visión de la tecnología. El imaginario colectivo es un indicador más, pues aparecen otras consideraciones que se refirieren a la incidencia de un dispositivo en educación.

A diferencia de otras pruebas proyectivas, no se tuvo en cuenta ni el tiempo que tardan, ni la reacción física, ni los silencios de los participantes, debido a que las evocaciones se vuelcan en un dispositivo que las recoge indiferenciadamente.

Esta valoración de grupo debatiendo e interpretando los resultados obtenidos individualmente, a falta de una interpretación inter-jueces (Sneiderman, 2011), añade 
validez y confiabilidad a la técnica. No se trata de que el investigador genere una única explicación del sentido de la película, sino que entre todos los participantes se le da forma.

Para concluir, esta técnica, como ocurre con los cuestionarios, tiene la particularidad de colocar a los participantes, al final del debate, en las contradicciones humanas de perseguir unos ideales y simultáneamente caer en la cuenta de practicar otros. Esta posición crítica tiene un valor formativo enorme para posteriores actividades formativas con los maestros.

Los participantes de la muestra intencional fueran grupos de estudiantes de la docencia regular en las titulaciones de Maestro de Educación primaria del curso académico 18-19 y Maestría de TIC en educación y formación del curso académico 19-20 de la Universidad Autónoma de Madrid (UAM). Mientras que el grupo de estudiantes de la titulación de Maestro de Educación primaria son estudiantes de primer año, con sus ilusiones y las particularidades de la selectividad que realiza la universidad (en esta con una nota media de acceso algo superior al aprobado, y con condiciones especiales en las calificaciones de Lengua y Matemáticas en las pruebas de acceso), el grupo de estudiantes de la Maestría de TIC en educación y formación tiene un particular sesgo o inclinación por los ordenadores que le ha hecho elegir una especialización centrada en la tecnología educativa. En las tres sesiones de la técnica de grupo focal, se trabajó respetivamente con 24 y 29 estudiantes de Maestro de dos grupos de primer año del curso 18-19 y con 20 estudiantes de Maestría de un único grupo del curso 19-20, con un total de 73 estudiantes universitarios de ambos sexos (Tabla 1).

TABLA 1

Participantes en el estudio

\begin{tabular}{|c|c|c|c|c|c|c|c|}
\hline $\begin{array}{c}\text { ID } \\
\text { grupo }\end{array}$ & $\begin{array}{c}\text { Nro. } \\
\text { sesiones }\end{array}$ & $\begin{array}{c}\text { Nro. } \\
\text { estudiantes }\end{array}$ & $\begin{array}{c}\text { Nro. } \\
\text { películas }\end{array}$ & Duración & $\begin{array}{c}\text { Titulación } \\
\text { universitaria }\end{array}$ & $\begin{array}{c}\text { Curso } \\
\text { académico }\end{array}$ & Asignatura \\
\hline 102 & 1 & 24 & 15 & $2 \mathrm{~h}$ & $\begin{array}{c}\text { Maestro Ed. } \\
\text { Primaria }\end{array}$ & $18-19$ & $\begin{array}{c}\text { Tecnología } \\
\text { educativa } \\
\text { básica }\end{array}$ \\
\hline 112 & 1 & 29 & 14 & $2 \mathrm{~h}$ & $\begin{array}{c}\text { Maestro Ed. } \\
\text { Primaria }\end{array}$ & $18-19$ & $\begin{array}{c}\text { Tecnología } \\
\text { educativa } \\
\text { básica }\end{array}$ \\
\hline Máster & 1 & 20 & 17 & $2 \mathrm{~h}$ & Maestría TIC & $19-20$ & $\begin{array}{c}\text { Ética y } \\
\text { sociedad }\end{array}$ \\
\hline Total & $\mathbf{3}$ & $\mathbf{7 3}$ & & $\mathbf{6 ~ h}$ & &
\end{tabular}

En la sesión de presentación de la asignatura de tecnología educativa básica para los dos grupos de los futuros maestros y en la sesión de presentación de la asignatura de ética y sociedad para el grupo de los futuros tecnólogos educativos, 
se solicitó a estos estudiantes que evocaran y escribieran el nombre de una película comercial que asociara cada uno de los participantes al concepto de "tecnología». Se recogieron las opiniones mediante una red social, que se utilizó como una pizarra digital en la que apuntar las ideas surgidas. A continuación, en la misma sesión, se solicitó a los estudiantes que indicaran, a la vista de las películas que habían compartido, cuáles eran las películas más recordadas y por qué creían que así había ocurrido, valorando el argumento de los filmes y las temáticas de orden moral que suscitaban. El debate posterior a los relatos personales compartidos permitió generar una orientación que interpretara los resultados obtenidos del análisis narrativo.

Para poder afinar el sentido de las evocaciones, en los grupos del curso 18-19 se solicitó de forma complementaria que mencionaran películas para los conceptos de "ciencia» y "educación", con un procedimiento similar de evocación y debate posterior. La idea era situar las películas evocadas en espacios sobre cuyos valores se pudiera hablar.

Las películas indicadas en cada sesión realizada fueron ordenadas y clasificadas a partir del debate mantenido en los grupos. Algunas películas estaban con el nombre que reciben en otro país, por lo que hubo que unificar su denominación. En los grupos de maestros alguna película estaba mal etiquetada (por las diversas evocaciones propuestas).

Del proceso de debates de los grupos de estudiantes y por las consideraciones que se fueron realizando, se identificaron cinco grandes categorías de evocaciones del concepto de tecnología: "terribles consecuencias», "violenta», "humana», "salvadora» $\mathrm{y}$ «otra»; que han permitido re-codificar las películas seleccionadas, para agruparlas y generar un pequeño análisis cuantitativo-descriptivo que abriera paso a las interpretaciones de la narrativa de los grupos de participantes. Los resultados gráficos obtenidos de la codificación de las películas permiten ofrecer tan solo una tendencia, no tienen relevancia estadística, y son ilustrativos de la posición del imaginario de los futuros maestros y tecnólogos educativos en relación con los relatos propuestos y discutidos en las sesiones del estudio.

\section{ReSUltados Y Discusión}

La categoría más numerosa de evocaciones sobre la tecnología entre todos los grupos de estudiantes participantes (figuras 1, 2 y 3) fue la de «terribles consecuencias». Esta categoría recogió películas en las que la sociedad (en muchas ocasiones futura o distópica), vive la segregación (Elysium), sufre la pérdida de libertades (In time, Tiempos modernos) o la posibilidad de las personas para actuar libremente (Minority Report), porque padecen el control (El juego, El círculo), el espionaje mental (Origen) o el terror (Blade Runner, Matrix) que la tecnología porta o permite practicar a una minoría, o bien se viven situaciones donde las máquinas (que pueden ser robots) proponen nuevas instancias con dilemas morales que generan sus actos y 
que sufre la humanidad (Her, Inteligencia artificial), o bien están fuera de control y producen toda una serie de desgracias (Yo, robot).

Los relatos emergentes de la categoría "terribles consecuencias" guardan una fuerte relación con el relato del cientifismo. La primera imagen del pensamiento pedagógico cuando reflexiona sobre el papel de la tecnología educativa es de preocupación ante la idea de primacía a una forma de hacer ciencia y sus máquinas controlando el mundo. A partir de estos análisis, las terribles consecuencias se caracterizan a partir de los usos inadecuados de la tecnología que traspasan los intereses de la sociedad a favor de un pequeño grupo de poderosos.

En el extremo de las situaciones distópicas de «terribles consecuencias» está la categoría de "violencia», un número mucho más pequeño de evocaciones sobre la tecnología, que prácticamente desaparece entre el grupo de los tecnólogos educativos (figura 3). En esta categoría, las películas mencionadas evocaban la tecnología como la herramienta que propicia la violencia o bajo la que surge gratuitamente la violencia (Transformers).

La categoría "violencia" generó una narrativa asociada al relato totalitario del maquinismo, que desea convertir a las personas en parte de su engranaje. Un estado anti-humado de permanente vigilancia que desemboca, finalmente, en un estado de ánimo contra las máquinas. Se trata de un relato terrorífico que trabaja desde la guerra y la violencia para imponerse como razón de ser. En los imaginarios de este relato maquinista, las tecnologías son los enemigos contra quienes combatir.

FIGURA 1

Tipos de imaginarios para el concepto «tecnología» en el grupo «102»

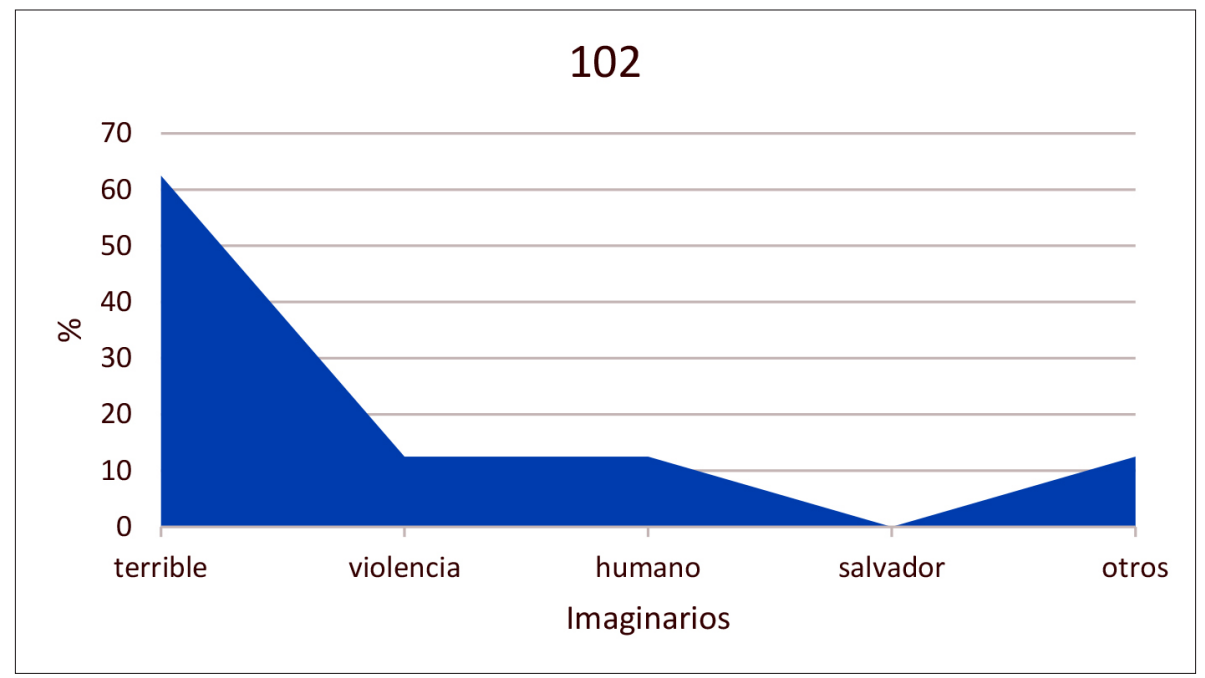


JOAQUÍN PAREDES-LABRA Y ADA FREITAS CORTINA

LAS REPRESENTACIONES DE LOS FUTUROS PROFESORES SOBRE LOS USOS

DE LA TECNOLOGÍA EN LA ESCUELA. UN ESTUDIO NARRATIVO

FIGURA 2

Tipos de imaginarios para el concepto «tecnología» en el grupo «112»

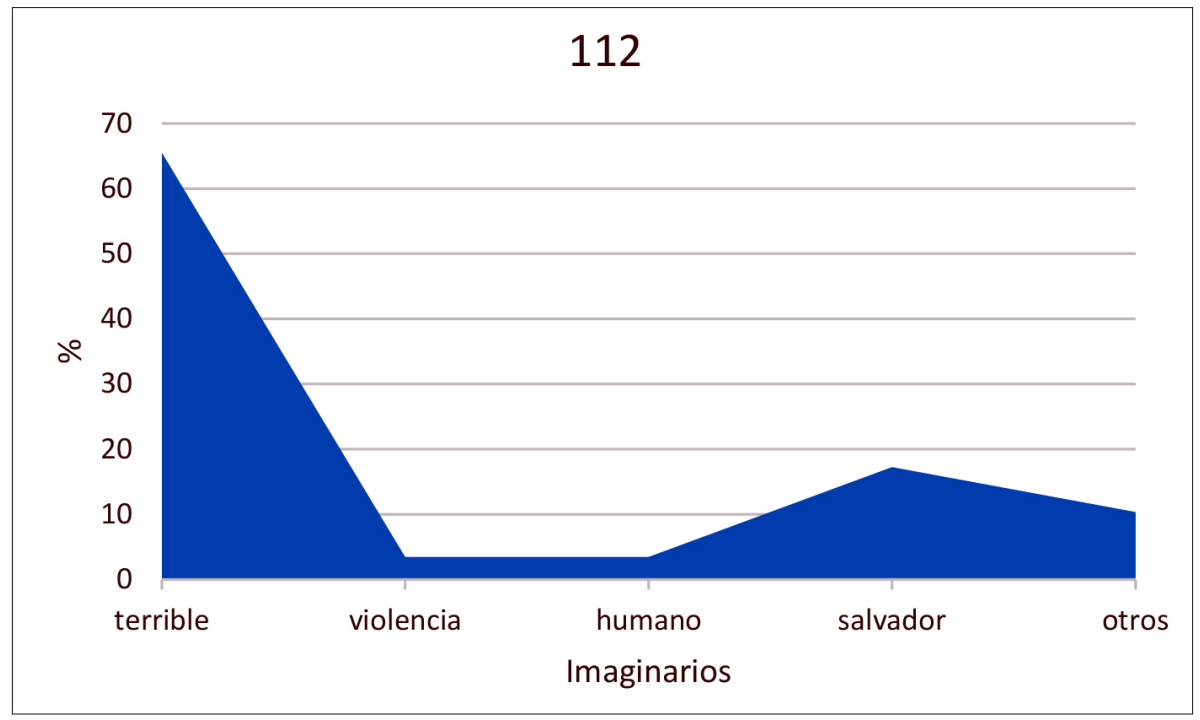

FIGURA 3

Tipos de imaginarios para el concepto «tecnología» en el grupo «máster»

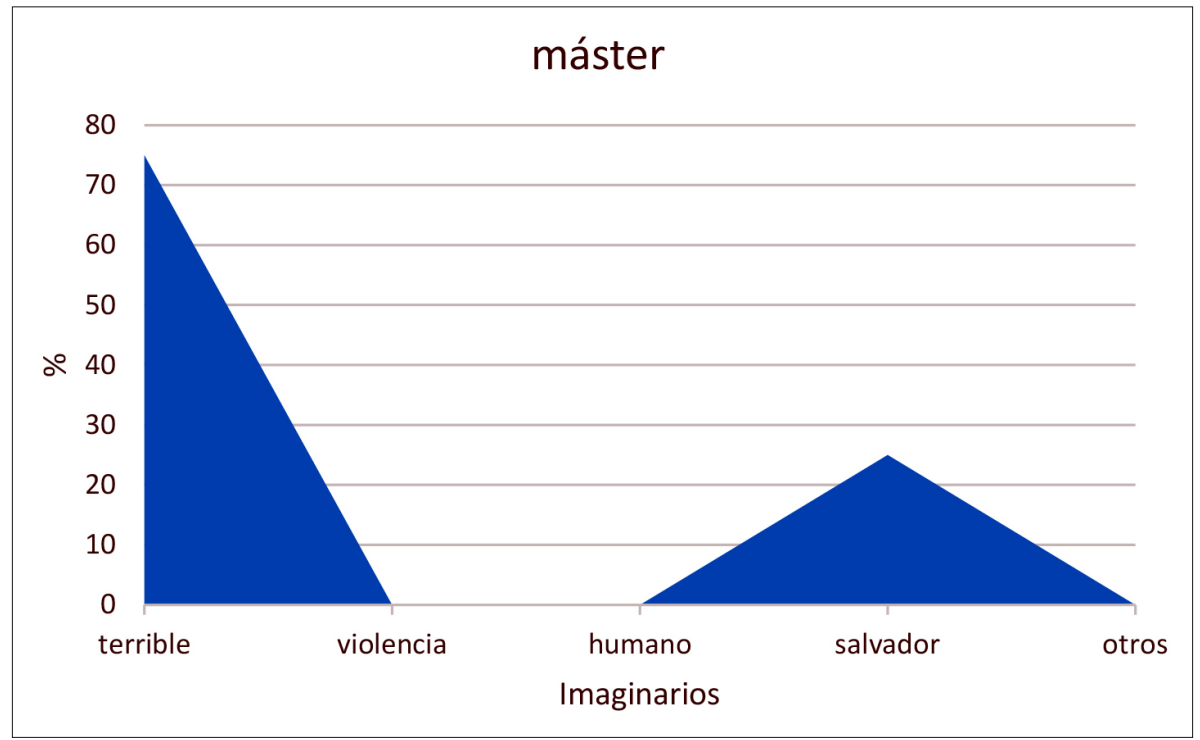


JOAQUÍN PAREDES-LABRA Y ADA FREITAS CORTINA

LAS REPRESENTACIONES DE LOS FUTUROS PROFESORES SOBRE LOS USOS DE LA TECNOLOGÍA EN LA ESCUELA. UN ESTUDIO NARRATIVO

Por otra parte, han sido mínimas las evocaciones de la tecnología en la categoría "humano", donde las máquinas, que son androides o robots con rasgos humanos, encarnan los mejores valores humanos (WALL $\bullet$ E, Chappie). Por tanto, los relatos emergentes de la categoría "humano", asociada al concepto de tecnología, se caracterizan con los imaginarios del relato humanista, donde lo tecnológico está profundamente cruzado con el acervo cultural humano y utiliza ese conocimiento fundamental para resolver multitud de dificultades y aplicar soluciones sostenibles.

Asimismo, la segunda categoría más relevante de evocaciones sobre la tecnología entre todos los grupos de estudiantes participantes fue la de «salvador», especialmente destacada en el grupo de futuros tecnólogos educativos (figura 3). La tecnología como salvadora es usada para ayudar a "los buenos" (Misión Imposible), a un país (Pixels), al curso de la historia (Regreso al futuro) o a la humanidad en su conjunto (Interstellar).

La categoría "salvador» generó una narrativa sobre el papel de la tecnología que se asocia al relato del futurismo, por su capacidad de garantizar las posibilidades de justicia social y de bienestar de la humanidad. En esta visión casi utópica, la tecnología se usa como herramienta de acción político-social en la defensa, protección y reivindicación de los derechos universales de los colectivos específicos y las minorías desfavorecidas para la superación de las dificultades de accesibilidad, igualdad, pacificación, etc.

Por último, la categoría "otros" hace referencia a otros temas específicos de la aplicación instrumental de la tecnología que no se asocian a los rasgos de ninguno relato popular en concreto, como es la evocación de la descripción en biopics del origen de determinado software o red social (Facebook), la alusión a algún juego transmedia (Tron) o la contribución de la tecnología a la magia o el entretenimiento (Abora me ves). Estos relatos estériles de valor moral podrían representar, en definitiva, la alienación de estos estudiantes, que están sometidos al vacío argumental de la necesidad de consumo de tecnología.

Por lo tanto, se evidencia una visión de tecnología marcadamente pesimista, que contrasta con la visión de valores renovadores, confianza en las personas, reto y superación que obtuvieron las evocaciones de las películas que representaron el imaginario de la educación de los futuros maestros, fruto del resultado de la actividad complementaria de evocación sobre «educación» aplicada a los grupos del curso 18-19. En el debate con los grupos (102 y 112) de futuros maestros surge que las películas que eligen como modelo educativo, aun estando adobadas por violencia (La ola), drogas, marginalidad o pobreza (Camino a la escuela, Los chicos del coro) y exacerbando las dificultades de los jóvenes, finalmente se alcanzan altos fines personales. La mayoría, si no todas las evocaciones sobre la educación, tienen el matiz de contexto juvenil (ajeno a la primera infancia con la que van a trabajar estos futuros profesionales). Pero son historias de superación, con final feliz, que representan cómo se perciben a sí mismos los futuros docentes ante la labor de la educación. 
Por su parte, para los futuros tecnólogos educativos (con algunos años más de edad), el imaginario de la educación se traduce en palabras como "transformar", "crear» o "progresar", que en cierta forma coincide con la parte final de las evocaciones del imaginario de sus compañeros más jóvenes, con la misma idea de superación. Las «terribles consecuencias» de las aplicaciones de corte cientifista de la tecnología pesan enormemente entre los estudiantes. Esta desconfianza contrasta con la esperanza, manifestada por un pequeño número de futuros tecnólogos educativos, de un futuro de transformación y cambio en el que la tecnología "salve» a la humanidad, en el sentido que le otorgan a la educación, de superar sus problemas y limitaciones. Esta perspectiva futurista, aunque minoritaria entre los estudiantes, hace abrigar alguna esperanza a un diálogo crítico con la tecnología educativa.

En general, por tanto, en el punto de partida de estas materias donde se recogen estas evocaciones se observa que hay poco margen para armar un relato alternativo para la tecnología que no repugne a quienes padecen las consecuencias de su preeminencia o sus servicios a la dominación.

Sin embargo, hay cierta ingenuidad, esta vez de parte de los detractores de la tecnología, al pensar que no forma parte de los procesos de producción de la cultura contemporánea. El propio cine y otras manifestaciones en los medios de comunicación, la literatura o el arte muestran la importancia del software, dispositivos portables o instalaciones con tecnología para generar su mensaje. Todos los imaginarios posibles están mediados por la tecnología. No se pretende justificar con ello que haya que adoptar irreflexivamente la tecnología. No es un problema de imperativo técnico (Sancho, 1998). Pero sí de armar un diálogo con la tecnología como el que ya organizaron las artes y la cultura.

Sería necesario construir un pensamiento alternativo a los imaginarios ya presentados, con más propuestas de reflexión en el enfoque ciencia-tecnología-sociedad, en la línea de la ética informacional de Luciano Floridi, para dar respuesta a las preocupaciones éticas que despierta el movimiento del «transhumanismo» en el relato cientifista dominante. Hace falta un humanismo de la gente corriente, que utilice un recurso esencial para estar en el mundo. Desgraciadamente, «ese público suele no estar al corriente de lo que puede hacerse con la tecnología» (Molinuevo, 2004, p. 122). Mucho menos si casi la mitad de las universidades españolas no incluyen en la formación de maestros estudios obligatorios sobre tecnología educativa.

Ya han existido posiciones de tecnología con propósito educativo con visiones humanistas y de corte político, por mundanas y concernidas, tal y como representa el pensamiento de Seymour Papert (1980) y la programación como estrategia para comprender el mundo, Laurence Lessig (2006) y la generación de conocimiento para compartir con todos gracias a un sistema de licencias o Richard Stallman (2002) y el software libre como lengua común de muchos proyectos. Son ejemplos de lo que se planteó al principio como «escuela tecnológica» desde un enfoque humanista, 
basada en problemas que afectan a la ciudadanía y para los que se buscan soluciones tecnológicas.

Asimismo, hay un imaginario de la ciencia en la escuela, pacifista y comprometido, como el del maestro republicano de la película La lengua de las mariposas, dirigida por José Luis Cuerda (Romea, 2011), y en muchos proyectos colaborativos en la web (Fernández Díaz y Correa, 2007).

Por lo tanto, cabría acercar el uso de la tecnología educativa hacia propuestas que estén vinculadas a los rasgos del ideario humanista, de una identidad digital indisociable de la cultura, donde la tecnología sea una herramienta idónea para despertar el interés por la producción de conocimiento crítico, dar soluciones a proyectos sociales y defender los ideales humanos.

\section{Conclusión}

A partir de los análisis de enfoque narrativo, se evidenció que los relatos emergentes de la tecnología entre futuros docentes se correspondían, de forma casi directa, con alguna de las cuatro grandes corrientes de pensamiento asociadas al papel de la tecnología educativa. Estos relatos, de menor a mayor presencia, son: el asociado a la categoría de evocaciones a la "violencia", que se asocia al relato totalitario del maquinismo; el de la categoría de evocaciones al «humano» (de las máquinas), próximo al relato del humanismo; el de la categoría de evocaciones como elemento "salvador", vinculado con el relato del futurismo; y, como más descollante, la categoría de evocaciones a las «terribles consecuencias», que se asocia al relato distópico del cientifismo.

El relato subyacente marcadamente pesimista sobre el papel de la tecnología en la sociedad, presente en el imaginario de los futuros maestros y futuros profesionales de la educación, hace plantearse qué podría pasar para que haya un relato humanista de la tecnología educativa y para que el docente, con ayuda de las TIC (de una escuela tecnológica), fuera simultáneamente humanista, cientifista crítico y futurista.

Podría haber una escuela tecnológica con rasgos humanistas cuando los docentes ponen en marcha principios para esa escuela con tales rasgos, como se planteó en la introducción. Así, deciden que les gusta la gente del entorno, no solo los niños, y utilizan la tecnología en la escuela para conectarla con la comunidad (principio de incidencia en la sociedad). O viven en el mundo que les toca, y no reniegan de él, abordando problemas cotidianos (principio de cotidianeidad). Consumen noticias, las analizan para sí y toman posición, haciendo actual lo que se aborda en las clases. Son renacentistas y futuristas, preocupados por la lectura, la interdisciplinariedad y el pensamiento libre. Se comprometen políticamente, con la gente, y éticamente, con unos valores, siendo más honestos y concernidos con las luchas en las que se involucra la comunidad (principio político). Dan dimensión humana a la escuela en la que trabajan, utilizan la tecnología para no vivir de espaldas a los niños ni a la 
comunidad. Vigilan las posiciones maquinistas, que tientan con sus productos tecnológicos a todas las escuelas. Luchan por la paz, contra el cambio climático, contra el consumismo, y lo denuncian con los medios tecnológicos de que disponen, por sí y con la escuela (principio ecológico). Se posicionan contra el cientifismo como nuevo escapismo o supremacismo. Y, por el contrario, construyen conocimiento, una narración con sus estudiantes de su mundo, con sus amenazas y miedos (principio de búsqueda del conocimiento). El conocimiento no debería ser simplemente el currículo ni «el día de X». Aprovechan el futurismo para construir utopías y cada proyecto con sus estudiantes es un desafío. Son críticos con la sociedad que les toca vivir. Se asocian. Enseñan y fomentan la participación con tecnología.

En definitiva, como se planteó al caracterizar una escuela tecnológica y al observar las derivas de la tecnología en algunos relatos, una solución futurista, utópica y participativa con tecnología es posible en las escuelas que emprenden proyectos transformadores de una realidad local o vinculados a otras escuelas de otras regiones y países (principio de colaboración). Se trata de una forma de entender la educación con tecnología en la comunidad, desde una visión ampliada de derechos y deberes de los ciudadanos.

Este renovado relato podría ser construido a partir de los principios del enfoque humanista (incidencia en la sociedad, cotidianeidad, político, ecológico, búsqueda conocimiento, colaboración), pero hará falta que los centros de formación de maestros y las escuelas se pongan en marcha para hacerlo. Y tomar conciencia de los imaginarios involucrados en la caracterización de la presencia de la tecnología en cada entorno educativo será el primer paso. Particularmente porque hará falta que las propias instituciones de formación inicial adopten metodologías con una práctica coherente (Fraga-Varela y Rodríguez-Groba, 2017) con los relatos propugnados.

\section{REFERENCIAS BIBLIOGRÁFICAS}

Adell, J., Castañeda, L., y Esteve, F. (2018). ¿Hacia la Ubersidad? Conflictos y contradicciones de la universidad digital. RIED. Revista Iberoamericana de Educación a Distancia, 21(2), 51-68. https://doi.org/10.5944/ried.21.2.20669.

Aranda, D., Creus, A., y Sánchez-Navarro, J. (2013). Educación, medios digitales y cultura de la participación. Barcelona: Uoc.

Area, M. (1991). Los medios, los profesores y el currículo. Hospitalet de Llobregat, Barcelona: Sendai.

Area, M. (2008). La innovación pedagógica con TIC y el desarrollo de las competencias informacionales y digitales. Investigación en la escuela, 64, 5-18.

Baram, M. (1971). Social Control of Science and Technology. Science, 172(3983), 535-539. https://doi.org/10.1126/science.172.3983.535.

Bautista, A. (1996). Posibles funciones de las nuevas tecnologías en la enseñanza en vísperas del cambio de milenio. Revista Complutense de Educación, 7(1), 121-130. 
JOAQUÍN PAREDES-LABRA Y ADA FREITAS CORTINA

LAS REPRESENTACIONES DE LOS FUTUROS PROFESORES SOBRE LOS USOS DE LA TECNOLOGÍA EN LA ESCUELA. UN ESTUDIO NARRATIVO

Bernal, F. (2016). Corporativismo y fascismo. Los sistemas de relaciones laborales autoritarios en la Europa de entreguerras. Hispania Nova. Revista de Historia Contemporánea, 15, 1-12. Bonfill Batalla, G. (1987). El México profundo, una civilización negada. México: Grijalbo.

Bruce, B., Peterson Bishop, A., y Budhathoki, N. (Eds.). (2014). Youth Community Inquiry: New Media for Community and Personal Growth. New York: Peter Lang.

Cabero, J. (2001). Tecnología educativa: diseño y utilización de medios en la enseñanza. Barcelona: Paidós.

Castañeda, L., Gutiérrez, I., y Rodríguez Cifuentes, M. T. (2011). El trabajo colaborativo mediado por las tecnologías. En M. Cebrián, M. J. Gallego Arrufat (Coords.), Procesos educativos con TIC en la sociedad del conocimiento (pp. 191-198). Madrid: Pirámide.

Castells, M. (1997-2005). La era de la información: economía, sociedad y cultura. Madrid: Alianza editorial.

Castoriadis, C. (1975). La institución imaginaria de la sociedad. Barcelona: Tusquets.

Cebrián, J. L. (1998). La red. Madrid: Taurus.

Cerbino, M., y Belotti, F. (2016). Community Media as Exercise of Communicative Citizenship: Experiences from Argentina and Ecuador. [Medios comunitarios como ejercicio de ciudadanía comunicativa: experiencias desde Argentina y Ecuador]. Comunicar, 47, 49-56. https://doi.org/10.3916/C47-2016-05.

Claramonte, J. (2012). Estéticas y Políticas del Nazismo. Espacio, Tiempo y Forma, 25, 333-344.

Da Rosa, G. A., y Trevisan, A. (2016). Filosofia da tecnologia e educação: conservação ou crítica inovadora da modernidade? Avaliação (Campinas, Brasil), 21(3), 719-737. https:// doi.org/10.1590/S1414-40772016000300004.

Escudero, J. M. (Dir.). (1989). Evaluación del proyecto Atenea. Informe de Progreso. Programa de Nuevas Tecnologías de la Información y Comunicación. Madrid: Ministerio de Educación y Ciencia.

Fernández Díaz, E. y Correa, J. M. (2008). Integración de las TIC en proyectos colaborativos mediante apadrinamientos digitales. RELATEC: Revista Latinoamericana de Tecnología Educativa, 7(2), 57-67.

Floridi, L. (2006). Ética de la información: su naturaleza y alcance. Isegoría: Revista de filosofía moral y política, 34, 19-46.

Fraga-Varela, F., y Rodríguez-Groba, A. (2017). Dilemas y desafíos de la Tecnología Educativa en el EEES: percepciones y creencias de futuros maestros. Profesorado, 21(1), 123-142.

Fullan, M., Watson, N., y Anderson, S. (2013). Ceibal. Próximos pasos. Informe final. Recuperado de: https://es.slideshare.net/inicials/fullan-versionfinaltraduccioninformeceibal. (Consultado el 16/10/2019).

Garci, J. L. (1971). Ray Bradbury, humanista del futuro. Madrid: Helios.

Garrison, D., y Anderson, T. (2005). El e-learning en el siglo XXI. Barcelona: Octaedro.

Gergen, K. (1991). El yo saturado. Dilemas de identidad en el mundo contemporáneo. Barcelona-Buenos Aires-México: Paidós.

Gértrudix, M., Borges, E., y García García, F. (2017). Vidas registradas. Redes sociales y jóvenes en la era algorítmica. Telos: Cuadernos de comunicación e innovación, 107, 62-70. 
JOAQUÍN PAREDES-LABRA Y ADA FREITAS CORTINA

LAS REPRESENTACIONES DE LOS FUTUROS PROFESORES SOBRE LOS USOS

DE LA TECNOLOGÍA EN LA ESCUELA. UN ESTUDIO NARRATIVO

Giachi, S. (2017). La evolución de la gobernanza de las políticas de innovación en España: el caso de la colaboración entre ciencia e industria. Política y Gobernanza. Revista de Investigaciones y Análisis Político, 1, 109-132.

Haraway, D. (2016). Manifiesto para cyborgs. Ciencia, tecnología y feminismo socialista a finales del siglo XX. Madrid: Editorial puente aéreo.

Hernando Calvo, A. (2017). Viaje a la escuela del siglo XXI. Así trabajan los colegios más innovadores del mundo. Madrid, España: Fundación Telefónica.

Herrán, A., Paredes, J., Moral, C., y Muñoz, T. (Coords.). (2012). Preguntas fundamentales sobre la enseñanza. Madrid: Universitas.

Hobsbawm, E. J., y Rudé, G. (1969). Revolución industrial y revuelta agraria. Madrid: Siglo veintiuno.

Jarpa, C. (2015). Función política de la educación en el pensamiento de Antonio Gramsci. Cinta de Moebio: Revista Electrónica de Epistemología de Ciencias Sociales, 53. Recuperado de: https://cintademoebio.uchile.cl/index.php/CDM/article/view/36717/38298 (Consultado el 05/02/2020).

Duek, C., Benítez, S., y Moguillansky, M. (2017). Niños, nuevas tecnologías y género: hacia la definición de una agenda de investigación. Fonseca, Journal of Communication, 14, 167-179. https://doi.org/10.14201/fjc201714167179.

Hernando Calvo, A. (2017). Viaje a la escuela del siglo XXI. Así trabajan los colegios más innovadores del mundo. Madrid: Fundación Telefónica.

Lessig, L. (2006). The Code 2.0. Cambridge: Basic Books.

Masuda, Y. (1984). La sociedad informatizada como sociedad post-industrial. Madrid: Fundesco-Tecnos.

Mateos, T., y Núñez, L. (2011). Narrativa y educación: indagar la experiencia escolar a través de los relatos. Teoría de la Educación. Revista Interuniversitaria, 23(2), 111-128.

Molinuevo, J. L. (2004). Humanismo y nuevas tecnologías. Madrid: Alianza.

Murcia, N. (2012). La escuela como imaginario social. Apuntes para una escuela dinámica. Magistro. Revista de la Maestría en Educación, 6(12), 53. https://doi.org/10.15332/ s2011-8643.2012.0012.03.

Navarro-Daniels, V., y Paukner, F. I. (2004). El proyecto social de Jacques Maritain. A Parte Rei: revista de filosofía, 33. Recuperado de: http://serbal.pntic.mec.es/ cmunoz1 (Consultado el 16/10/2019).

Ordóñez, J., Sánchez Ron, J. M., y Navarro, V. (2007). Historia de la ciencia. Pozuelo de Alarcón (Madrid): Espasa-Calpe.

Papert, S. (1980). Mindstorms: children, computers, and powerful ideas. Brighton:The Harvester Press.

Paredes, J., Guitert, M., y Rubia, B. (2015). La innovación y la tecnología educativa como base de la formación inicial del profesorado para la renovación de la enseñanza. RELATEC: Revista Latinoamericana de Tecnología Educativa, 14(1), 101-114.

Paredes, J., Freitas, A., y Andrés, C. (2017). La escuela que transformó el currículo con tecnología. Desafíos para sus docentes y la formación que necesitan. Revista e-Curriculum, 15(1), 4. https://doi.org/10.23925/1809-3876.2017v15i1p4-24.

Rico, F. (1993). El sueño del humanismo. Madrid: Alianza. 
JOAQUÍN PAREDES-LABRA Y ADA FREITAS CORTINA

LAS REPRESENTACIONES DE LOS FUTUROS PROFESORES SOBRE LOS USOS DE LA TECNOLOGÍA EN LA ESCUELA. UN ESTUDIO NARRATIVO

Ricoeur, P. (1995). Tiempo y narración. I: Configuración del tiempo en el relato bistórico México-Buenos Aires: Siglo XXI.

Rivas Sacconi, J. M. (1947). Miguel Antonio Caro, humanista. Bogotá: Instituto Caro y Cuervo.

Rodríguez de las Heras, A. (1991). Navegar por la información. Madrid: Fundación para el Desarrollo de la Función Social de las Comunicaciones.

Romea, C. (2001). Lectura a cinco bandas: «La lengua de las mariposas». Comunicar, 17, 71-78.

Salinas, J. (1998). Telemática y educación: expectativas y desafíos. Comunicación y Pedagogía: nuevas tecnologías y recursos didácticos, 151, 8-16.

Salinas, J. (1999). Uso educativo de las redes informáticas. Educar, 25, 81-92.

Sánchez-Antolín, P., Ramos, F. J., y Sánchez Santamaría, J. (2014). Formación continua y competencia digital docente: el caso de la Comunidad de Madrid. Revista Iberoamericana de Educación, 65, 91-110.

Sancho, J. M., y Hernández Hernández, F. (2016). Que no cambiemos para que todo permanezca igual. Dosier Graó, 1, 8-13.

Sancho, J. M. (Coord.). (1998). Para una tecnología educativa. Barcelona: Horsori.

Sancho, J. M. (2018). Innovación y enseñanza. De la "moda» de innovar a la transformación de la práctica docente. Educação, 41(1), 12. https://doi.org/10.15448/1981-2582.2018.1.29523.

Saura, G., Muñoz-Moreno, J., Luengo-Navas, J., y Martos-Ortega, J. (2017). Protesting on Twitter: Citizenship and Empowerment from Public Education. [Protestando en Twitter: ciudadanía y empoderamiento desde la educación pública]. Comunicar, 53, 39-48. https://doi.org/10.3916/C53-2017-04.

Senabre, E., Ferran-Ferrer, N., y Perelló, J. (2018). Participatory design of citizen science experiments. [Diseño participativo de experimentos de ciencia ciudadana]. Comunicar, 54, 29-38. https://doi.org/10.3916/C54-2018-03.

Sneiderman, S. (2011). Consideraciones acerca de la confiabilidad y validez en las técnicas proyectivas. Revista Subjetividad y Procesos Cognitivos, 15(2), 93-110.

Stallman, R. (2002). Free software, free society: selected essays of Richard M. Stallman. Boston: Free Software Foundation.

Trejo, R. (1996). La nueva alfombra mágica: usos y mitos de Internet, la red de redes. Madrid: Fundesco.

Wenger, E. (1998). Communities of Practice: Learning, Meaning, and Identity. Learning in Doing: Social, Cognitive and Computational Perspectives. New York City: Cambridge University Press. 